\title{
An iterative ab initio non-Hermitian Floquet method for photoionization resonances
}

\author{
Y. Sajeev \\ Theoretical Chemistry Section, Bhabha Atomic Research Centre, Mumbai 400 085, India.
}

\begin{abstract}
We present an efficient $a b$ initio non-Hermitian Floquet method for computing the photoionization resonances of an electronic system interacting with linearly polarized monochromatic laser light. Unlike the direct "brute force" diagonalization method, which has been used for huge Floquet matrix eigenvalue problems, the new method follows a simple iterative process. The computational advantages of the iterative method are very remarkable as it avoids computation, storage, and diagonalization of the huge Floquet matrix. The new method can also be used in conjunction with the $a b$ initio computational techniques that were originally developed for the field-free bound state calculations. The method is best illustrated with the photoionization resonance of the hydrogen atom.
\end{abstract}

\section{Introduction}

The Floquet methods have been applied to a wide range of atomic and molecular multiphoton processes [1, 2, 3, 4]. The Floquet formalism allows the reduction of periodic or quasi-periodic time-dependent Schrödinger equation into a time independent matrix eigenvalue problem. The method is based on the use of Floquettype operator in an extended Hilbert space which was introduced by Sambe for the time-periodic Hamiltonian [5] and was extended by Howland for the general time-dependent Hamiltonian [6]. The spatial part of the composite Hilbert space is spanned by an orthogonal set of square-integrable basis functions and the temporal part is spanned by a complete orthogonal set of time-periodic functions. Direct 'brute force' diagonalization of the Floquet Hamiltonian represented in the extended Hilbert space is the most frequently used method for solving the Floquet eigenvalue problem. Another method for solving the time-dependent periodic and quasi-periodic problems is the $\left(t, t^{\prime}\right)$ formalism [7]. The $\left(t, t^{\prime}\right)$ formalism has 
the advantage of incorporating the Floquet methods in the existing computational frameworks that were originally developed for time-independent Hamiltonian [8]. In the $\left(t, t^{\prime}\right)$ formalism, $t^{\prime}$ acts as an additional time coordinate and the operator that defines the system interacting with the field, $H=H\left(r, t^{\prime}\right)-i \hbar \partial / \partial t^{\prime}$, is treated as a time-independent operator. The cost of computation for the direct diagonalization and the $\left(t, t^{\prime}\right)$ methods sharply increases with the number of spatial and temporal basis sets.

In this Communication, we present a new iterative method for calculating photoionization resonances of an electronic system interacting with a linearly polarized monochromatic field. The goal of the new method is to make use of the existing $a b$ initio bound state methodologies (i.e., using the $L^{2}$ basis set) in a computationally viable way for the photoionization resonance states which are embedded in the continuum. The analytical continuation of the Hamiltonian using complex scaling $[9,10,11,12,13]$ plays a fundamental role in our formulation of the new iterative procedure. In the complex scaling formalism, we have the advantage of treating the resonance state using the $L^{2}$ basis sets. The fundamental works by Balslev and Combes [9, 11] and by Simon [10] have provided the underlying mathematical foundations for this method. The complex scaled Hamiltonian is obtained when a dilation transformation $r \rightarrow r \eta$, using a complex scaling parameter $\eta=e^{i \theta}$, is applied for the electronic coordinates. The complex scaling transformation of the electronic coordinates results a non-Hermitian Hamiltonian and may have complex eigenvalue corresponding to the resonance state with a finite lifetime. A detailed description of the non-Hermitian formalism can be found in monographs and books that are devoted for the many-electron resonance problems $[12,13,14]$. The resonance wavefunctions become squareintegrable upon the complex scaling transformation. Thus, the complex scaling method has the fundamental advantage of associating a resonance state with a single wavefunction which is embedded in the generalized Hilbert space instead of a wavepacket (i.e., a collection of continuum eigenstates of the non-scaled Hermitian Hamiltonian). Within the complex scaling approach, the energy position $\left(E^{r e s}\right)$ and the width $(\Gamma)$ of a resonance are associated with the complex eigenvalues, $E^{\eta}=E^{r e s}-i \Gamma / 2$, of the complex scaled Hamiltonian. 


\section{Non-Hermitian Floquet method}

Here we discuss briefly the non-Hermitian Floquet formalism for the calculation of photoionization resonance, as being formulated by Chu and Reinhardt [15]. The complex scaled time-dependent Schrödinger equation for a system interacting with an external laser field is given by

$$
\left(H_{F F}^{(\eta)}(r)+V^{(\eta)}(r, t)\right) \Psi_{\alpha}^{(\eta)}(r, t)=i \hbar \frac{\partial}{\partial t} \Psi_{\alpha}^{(\eta)}(r, t),
$$

where $H_{F F}^{(\eta)}(r)$ is the complex scaled field-free Hamiltonian and $V^{(\eta)}(r, t)$ is the complex scaled time dependent operator corresponding to the light-matter interaction. The elctronic coordinate $r$ in the wavefunction $\Psi_{\alpha}^{(\eta)}(r, t)$ symbolizes all spatial and spin components. The complex scaled time-dependent operator which describes the interaction of an atom with a linearly polarized monochromatic laser field with frequency $\omega$ and maximum field amplitude $\varepsilon_{0}$ can be written in the length gauge using the dipole approximation as

$$
V^{(\eta)}(r, t)=\varepsilon_{0} D^{(\eta)}(r) \cos (\omega t)
$$

where $D^{(\eta)}(r)$ is the complex scaled electric dipole operator for an electric filed with polarization vector $e_{z}$, i.e.,

$$
D^{(\eta)}(r)=-\eta \sum_{i} e_{z} \cdot r_{i}
$$

According to the Floquet theorem, there are particular solutions $\Psi_{\alpha}^{(\eta)}(r, t)$ of equation (1) obeying an ansatz [16]

$$
\Psi_{\alpha}^{(\eta)}(r, t)=e^{-i \varepsilon_{\alpha}^{Q E} t / \hbar} \Phi_{\alpha}^{(\eta)}(r, t) .
$$

where $\Phi_{\alpha}^{(\eta)}(r, t)$ is time-periodic function with the period $T=2 \pi / \omega$ and $\varepsilon_{\alpha}^{Q E}$ is the complex quasienergy which gives the photoionization decay width $\Gamma_{\alpha}^{Q E}=$ $2 \operatorname{Im} \varepsilon_{\alpha}^{Q E}$. The complex scaled Floquet eigenenergy equation can be written as

$$
H_{f}^{(\eta)}(r, t) \Phi_{\alpha}^{(\eta)}(r, t)=\varepsilon_{\alpha}^{Q E} \Phi_{\alpha}^{(\eta)}(r, t),
$$

where $H_{f}^{(\eta)}(r, t)$ is the complex scaled Floquet Hamiltonian,

$$
H_{f}^{(\eta)}(r, t)=H_{F F}^{(\eta)}(r)+\varepsilon_{0} D^{(\eta)}(r) \cos (\omega t)-i \hbar \frac{\partial}{\partial t}
$$


and the time variable $t$ is considered as an additional coordinate. The basic problem in the calculation of photoionization resonance rate of decay is to construct the Floquet Hamiltonian in a given composite basis and to solve the eigenvalue equation (Eq. 5). In principle, this problem can be solved by conventional numerical techniques used for diagonalizing large complex symmetric matrices. However, we avoid this heavy computational task by developing an efficient iterative minimization formalism which is particularly suitable when we are interested in a particular resonance state which is associated to a uniquely defined fieldfree reference state. It should also be noted that the analytical continuation employed in the non-Hermitian Floquet method is by no means limited to the complex scaling method. The other widely used analytical continuation techniques such as complex absorbing potential methods and the continuum remover methods $[17,18,19,20,21]$ can also be used as the analytical continuation scheme in the general iterative procedure which will be discussed in the following section.

\section{The new iterative method}

We will begin our formal treatment of the iterative method by constructing a complete bi-orthogonal set of time-periodic functions $\{\chi(r, t)\}$ which spans the composite Hilbert space. The spatial part of the composite Hilbert space is spanned by the complete set of eigen solutions $\left\{\varphi_{i}^{(\eta)}(r)\right\}$ of the complex scaled field-free Hamiltonian,

$$
H_{F F}^{(\eta)}(r) \varphi_{i}^{(\eta)}(r)=E_{i}^{\eta} \varphi_{i}^{(\eta)}(r)
$$

and the temporal part is spanned by a complete orthogonal set of time-periodic functions $\left\{e^{i n \omega}\right\}$, i.e.,

$$
\begin{aligned}
& \chi_{i, n}^{(\eta)}(r, t)=\varphi_{i}^{(\eta)}(r) e^{i n \omega t} \\
& i=1,2, \ldots . N_{r}, \quad n=0, \pm 1, \pm 2 \ldots \pm N_{t}
\end{aligned}
$$

where $N_{r}$ is the total number of spatial basis functions and $M=2 N_{t}+1$ is the total number of time-periodic basis functions used. For the sake of clarity, we recall here that the eigenfunctions $\left\{\varphi_{i}^{(\eta)}(r)\right\}$ of a non-Hermitian Hamiltonian satisfy the orthonormality and closure relations only when the inner product definition is altered into c-product [22], i.e.,

$$
\left\langle\varphi_{j}^{(\eta) *}(r) \mid \varphi_{i}^{(\eta)}(r)\right\rangle=\delta_{i j}
$$


Having defined a complete bi-orthogonal set of time-periodic basis functions $\left\{\phi_{i}^{\eta}(r) e^{i n \omega}\right\}$, the exact wavefunction of the Floquet Hamiltonian defined in a given given basis set can now be expressed as,

$$
\left|\Phi_{\alpha}^{(\eta)}(r, t)\right\rangle=\sum_{j=1}^{N_{r}} \sum_{n=-N_{t}}^{+N_{t}} c_{j, n}^{\alpha}\left|\varphi_{j}^{(\eta)}(r)\right\rangle e^{i n \omega t}
$$

We now assume that one of the non-degenerate eigenfunctions of the field-free Hamiltonian, $H_{F F}^{(\eta)}(r)$, is a zeroth order approximation to the exact solution, $\left|\Phi_{\alpha}^{\eta}(r, t)\right\rangle$, of the complex scaled Floquet Hamiltonian, $H_{f}^{(\eta)}(r, t)$, that we are investigating. Furthermore, we shall also impose the intermediate normalization in the following (using the c-product rather than the usual scalar product), i.e.,

$$
\frac{1}{T} \int_{0}^{T} d t\left\langle\varphi_{\alpha}^{*}(\eta)(r) \mid \Phi_{\alpha}^{(\eta)}(r, t)\right\rangle=1
$$

This is well fulfilled in practice even for large field intensities. The exact solution, $\left|\Phi_{\alpha}^{\eta}(r, t)\right\rangle$, in the intermediate normalized form is obtained when $c_{\alpha, 0}^{\alpha}=1$ in equation (10). We now assume that the zeroth order function is known, and we proceed to find the coefficients for the remaining part of the exact wavefunction. In order to accomplish this, we return to the Floquet eigenvalue equation (Eq. 5), which we write as

$$
\left(H_{f}(r, t)-E_{\alpha}^{(\eta)}\right)\left|\Phi_{\alpha}^{(\eta)}(r, t)\right\rangle=\Delta \varepsilon_{\alpha}^{Q E(\eta)}\left|\Phi_{\alpha}^{(\eta)}(r, t)\right\rangle
$$

where

$$
\Delta \varepsilon_{\alpha}^{Q E(\eta)}=\varepsilon_{\alpha}^{Q E}-E_{\alpha}^{(\eta)}
$$

Operating by $\frac{1}{T} \int_{0}^{T} d t e^{-i \lambda \omega t}\left\langle\varphi_{k^{(\eta)}}^{*}(r)\right|$ from the left on to Eq. (12), we get the equations for the coefficients in the basis set expansion of $\left|\Phi_{\alpha}^{\eta}(r, t)\right\rangle$,

$$
\begin{aligned}
& \frac{1}{T} \int_{0}^{T} d t e^{-i \lambda \omega t}\left\langle\varphi_{k(\eta)}^{*}(r)\left|\left(H_{f}(r, t)-E_{\alpha}^{(\eta)}\right)\right| \Phi_{\alpha}^{(\eta)}(r, t)\right\rangle=c_{k, \lambda}^{\alpha} \Delta \varepsilon_{\alpha}^{Q E(\eta)} \\
& \lambda=0, \pm 1, \pm 2, \ldots \pm N_{t}, \quad k=1,2 \ldots, N_{r}
\end{aligned}
$$


Using Eq. (10), Eq. (14) becomes,

$$
\begin{gathered}
\frac{1}{T} \int_{0}^{T} d t e^{-i \lambda \omega t} \sum_{j=1}^{N_{r}} \sum_{n=-N_{t}}^{+N_{t}} c_{j, n}^{\alpha}\left\langle\varphi_{k(\eta)}^{*}(r)\left|H_{f}^{(\eta)}(r, t)-E_{\alpha}^{(\eta)}\right| \varphi_{j}^{(\eta)}(r)\right\rangle e^{i n \omega t} \\
=c_{k, \lambda}^{\alpha} \Delta \varepsilon_{\alpha}^{Q E(\eta)} .
\end{gathered}
$$

Using the fact that the field-free basis set used are bi-orthogonal, after integrating over the time coordinate, the left-hand-side of Eq. (15) can be written as

$$
\begin{aligned}
\frac{1}{T} \int_{0}^{T} d t e^{-i \lambda \omega t} \sum_{j=1}^{N_{r}} \sum_{n=-N_{t}}^{+N_{t}} c_{j, n}^{\alpha}\left\langle\varphi_{k(\eta)}^{*}(r)\left|H_{f}^{(\eta)}(r, t)-E_{\alpha}^{(\eta)}\right| \varphi_{j}^{(\eta)}(r)\right\rangle e^{i n \omega t} \\
=\sum_{j}^{N_{r}} \sum_{n=-N_{t}}^{+N_{t}}\left(\begin{array}{l}
c_{j, n}^{\alpha} E_{j}^{(\eta)} \delta_{k, j} \delta_{\lambda, n}+ \\
\frac{\varepsilon_{0}}{2} c_{j, n}^{\alpha}\left\langle\varphi_{k(\eta)}^{*}(r)\left|D^{(\eta)}(r)\right| \varphi_{j}^{(\eta)}(r)\right\rangle \delta_{(\lambda \pm 1), n}- \\
c_{j, n}^{\alpha} E_{\alpha}^{(\eta)} \delta_{k, j} \delta_{\lambda, n}+ \\
c_{j, n}^{\alpha} n \hbar \omega \delta_{k, j} \delta_{\lambda, n}
\end{array}\right),
\end{aligned}
$$

i.e., the diagonal elements of the matrix elements due to the left projection of basis set functions on to Eq. (12) equal the electronic field-free energies dressed by an integer number of photons and its off-diagonal elements are determined by couplings of dressed states that differ by exactly one photon. Using the simplifications employed in Eq. (16), the equations for the coefficients can now be written as

$$
\begin{aligned}
& \frac{\varepsilon_{0}\left(\sum_{j=1}^{N_{r}}\left(c_{j, \lambda+1}^{\alpha}+c_{j, \lambda-1}^{\alpha}\right)\left\langle\varphi_{k(\eta)}^{*}(r)\left|D^{(\eta)}(r)\right| \varphi_{j}^{(\eta)}(r)\right\rangle\right)}{2\left(\Delta \varepsilon_{\alpha}^{Q E(\eta)}-E_{k}^{(\eta)}+E_{\alpha}^{(\eta)}-\lambda \hbar \omega\right)}=c_{k, \lambda}^{\alpha} \\
& \lambda=0, \pm 1, \pm 2, \ldots \pm N_{t}, \quad k=1,2 \ldots, N_{r} .
\end{aligned}
$$

Since $\left|\Phi_{\alpha}^{\eta}(r, t)\right\rangle$ is intermediately normalized (i.e., $c_{\alpha, 0}^{\alpha}=1$ ), the equation for $c_{\alpha, 0}^{\alpha}$ serves as an equation for the $\Delta \varepsilon_{\alpha}^{Q E(\eta)}$, i.e.,

$$
\sum_{j=1}^{N_{r}} \frac{\varepsilon_{0}}{2}\left(c_{j, 1}^{\alpha}+c_{j,-1}^{\alpha}\right)\left\langle\varphi_{\alpha(\eta)}^{*}(r)\left|D^{(\eta)}(r)\right| \varphi_{j}^{(\eta)}(r)\right\rangle=\Delta \varepsilon_{\alpha}^{Q E(\eta)}
$$

As one can see from Eq. (18), $\Delta \varepsilon_{\alpha^{Q E(\eta)}}$ is solely determined by the coefficients of the Fourier functions containing one photon $(n \pm 1)$, i.e., $c_{j, 1}^{\alpha}$, and $c_{j,-1}^{\alpha}$. However, 
the equation for the coefficients $c_{j, 1}^{\alpha}$, and $c_{j,-1}^{\alpha}$ contains and, hence, couples with the coefficients of the Fourier components that differ by one photon, as being shown in Eq. (17). At the end of this derivation we have a hierarchy of equations that must be solved simultaneously to obtain the $\Delta \varepsilon_{\alpha}^{Q E(\eta)}$. These sets of equations are extremely large if a large number of field-free basis or Fourier-basis functions are used.

Below we discuss the important steps involved in writing an efficient computer program for solving the equations for quasienergy and the expansion coefficients. Computational efficiency can be achieved by formulating each of the equations as matrix-matrix or matrix-vector products, for which the modern computers are particularly adopted. For example, the set of coefficients can be stored as a matrix with elements $C_{j, \nu}=c_{j,\left(N_{t}+1-\nu\right)}$, where $c$ is the coefficient from eq. 10 and the row and column indices ( $j$ and $\nu$ ) are assigned for filed-free electronic basis set and the the Fourier basis functions, respectively.

$\mathbf{C}=\left(\begin{array}{ccccccccc}c_{1, N_{t}}^{\alpha} & c_{1, N_{t}-1}^{\alpha} & \cdots & c_{1,1}^{\alpha} & c_{1,0}^{\alpha} & c_{1,-1}^{\alpha} & \cdots & c_{1,-\left(N_{t}-1\right)}^{\alpha} & c_{1,-N_{t}}^{\alpha} \\ c_{2, N_{t}}^{\alpha} & c_{1, N_{t}-1}^{\alpha} & \cdots & c_{2,1}^{\alpha} & c_{2,0}^{\alpha} & c_{2,-1}^{\alpha} & \cdots & c_{2,-\left(N_{t}-1\right)}^{\alpha} & c_{2,-N_{t}}^{\alpha} \\ \vdots & \vdots & \vdots & \vdots & \vdots & \vdots & \vdots & \vdots & \vdots \\ c_{N_{r}, N_{t}}^{\alpha} & c_{N_{r}, N_{t}-1}^{\alpha} & \cdots & c_{N_{r}, 1}^{\alpha} & c_{N_{r}, 0}^{\alpha} & c_{N_{r},-1}^{\alpha} & \cdots & c_{2,-\left(N_{t}-1\right)}^{\alpha} & c_{N_{r},-N_{t}}^{\alpha}\end{array}\right)$

Similarly, the left-hand-side of Eq. (17) could be represented as product of a dipole matrix and a modified coefficient matrix. The contraction between these matrices given explicitly in Eq. (17) could be then written as a multiplication between the dipole matrix $\mathbf{D}$ and modified coefficient matrix $\mathbf{B}$ to produce the coefficient matrix:

$$
\mathbf{C}=\mathbf{D B}\left(\mathbf{C}, \Delta \varepsilon_{\alpha}^{Q E(\eta)}\right)
$$

where the individual element of the $\mathbf{D}$ and $\mathbf{B}$ matrices may be denoted as

$$
\mathrm{D}_{i, j}=\left\langle\varphi_{i}^{(\eta)^{*}}(r)\left|D^{(\eta)}(r)\right| \varphi_{j}^{(\eta)}(r)\right\rangle
$$

with

$$
B_{j, \nu}=\frac{\varepsilon_{0}}{2} \frac{C_{j, \nu+1}^{\alpha}+C_{j, \nu-1}^{\alpha}}{\Delta \varepsilon_{\alpha}^{Q E(\eta)}-E_{j}^{(\eta)}+E_{\alpha}^{(\eta)}-\left(N_{t}+1-\nu\right) \hbar \omega}
$$

The matrix equation (Eq. 20) can be solved by iterative methods. The initial guess may be inserted into the right-hand-side of Eq. (20) and subsequently used to get 
new coefficients and $\Delta \varepsilon_{\alpha}^{Q E(\eta)}$,

$$
\mathbf{C}_{i t n}=\mathbf{D B}\left(\mathbf{C}_{i t n-1}, \Delta \varepsilon_{\alpha}^{Q E(\eta)}{ }_{i t n-1}\right)
$$

where $i t n$ denotes the iteration number. For example, in the first iteration $\Delta \varepsilon_{\alpha}^{Q E(\eta)}$ is kept to zero and the coefficients are also taken to be zero except for $c_{\alpha, 0}^{\alpha}$. In order to keep exact wavefunction to be intermediately normalized with the zeroth order wavefunction, the coefficient $c_{\alpha, 0}^{\alpha}$ is always kept unity. The iterative process is continued until convergence is reached for $\Delta \varepsilon_{\alpha}^{Q E(\eta)}$ and for the coefficients. A simplified schematic representation of the computational algorithm is given in figure (1).

\section{Numerical example}

As a test case for the iterative procedure developed above, the quasienergy associated with the ground state of the field-free hydrogen atom were calculated. The complex scaled field-free eigen solutions of the hydrogen atom were calculated using the diagonalization of the complex scaled field-free Hamiltonian in a d-aug-pV6Z basis set. The calculations were done without the $h$ type basis function of the d-aug-pV6Z basis set. The field-free eigen solutions were used as a basis for the iterative calculation for the non-Hermitian Floquet Hamiltonian.

In our calculations for the quasienergy, the maximum field amplitude is held fixed at $\varepsilon_{0}=0.025 \mathrm{a} . u\left(I=2.1934 \times 10^{13} \mathrm{~W} / \mathrm{cm}^{2}\right)$ and the laser frequencies are varied for $0.5 a . u . \leq \omega \leq 0.7 a . u$. The quasienergy calculations were performed using 175 field-free basis sets and 21 Fourier basis functions $\left(N_{t}=10\right)$. The convergence of both the wavefunction (see Eq. (10)) and the quasi energy (see Eq. (18)) from iterative method are very rapid and only less than twenty iterations were enough for obtaining micro-hartree accuracy. The convergence of the quasienergy alone is even much faster as it depends only on the the convergence of the coefficients $c_{j, 1}^{\alpha}$ and $c_{j,-1}^{\alpha}$ (see Eq. (18)). Since $c_{j, 1}^{\alpha}$ and $c_{j,-1}^{\alpha}$ directly coupled to $c_{\alpha, 0}^{\alpha}$ (i.e., the coefficient of the field free state of interest), their convergence is very fast. In our numerical calculation, these coefficients and, hence, the quasi energy always converge in less than 6 iterations. The resonance position and width were obtained from the cusp in the $\eta$-trajectory of the complex quasi-energy [23]. The position and the rate of decay of the resonance state corresponding to the field perturbed ground state are shown in figures (2) and (3) respectively. The results obtained by using our new method are in complete agreement in all significant digits with the results that were obtained from the straightforward diagonalization of the non-Hermitian Floquet Hamiltonian when the same basis set has been used. 


\section{Concluding remarks}

The new iterative non-Hermitian Floquet method is formally very simple and straightforward to implement. The eigenvalues and eigenvectors associated with a particular field-free reference state is computed without storing large Floquet Hamiltonian matrix and without doing the diagonalization which scales the cost of computation as $N^{3}$, where $N=N_{r}(2 N t+1)$ is the total number of composite basis set used. If $\mathrm{N}$ is larger than several thousands, its diagonalization becomes computationally prohibitive. Our iterative method requires only the multiplication of two small matrices, i.e., the multiplication of the square matrix $\mathbf{D}$ of size $N_{r}$ with the matrix B of size $N_{r}-b y-\left(2 N_{t}+1\right)$. As we have illustrated, however, the matrix $\mathbf{B}$ depends on the unknown exact energy. Hence, self-consistency procedure is required. This limits the application of the method to treating just one energy level in one computation. However, it is not a serious limitation. In most practical applications (harmonic generation, above threshold ionization), the laser pulses are selected to populate only one photoinduced resonance state [24]. Since the eigenfunction for the resonance state are also computed in the iterative procedure, it can also be used for the ab initio calculation of the high harmonic generation spectra [8]. The iterative non-Hermitian Floquet methods can also be used in conjunction with the ab initio electronic structure methods, which have been conventionally used for computing the Field-Free eigen solutions and properties, for solving the photoionization resonances. Interestingly, an ab initio multireference configuration interaction method for the Hermitian Floquet problem has been recently discussed in the literature [25, 26, 27]. The iterative procedure can also be used for improving the computational efficiency of such a Floquet formalism. Finally, there is also a close analogy between the post Hartree-Fock $a b$ initio electron correlation calculation of the time-independent formalism and the iterative method developed in this Communication. In the ab initio electron correlation formalism, the analogous quantity $\Delta \varepsilon$ is often referred to as the correlation energy due to the coupling of the mean-field solutions by the two-electron operator used in the post Hartree-Fock methods [28]. However in our case, $\Delta \varepsilon_{\alpha}^{Q E(\eta)}$ arises due to the dipole coupling introduced in the Floquet method for the fieldfree solutions.

\section{Acknowledgments}

The author is grateful to Prof. Nimrod Moiseyev and Dr. Milan Sindelka for many stimulating discussions and suggestions. 


\section{References}

[1] S.-I. Chu, Adv. Chem. Phys. 73 (2007) 739.

[2] S.-I. Chu, D. A. Telnov, Phys. Rep. 390 (2004) 1.

[3] M. Dorr, M. Terao-Dunseath, P. G. Burke, C. J. Joachain, C. J. Noble, J. Purvis, J. Phys. B 28 (1995) 3545.

[4] N. Moiseyev, F. Weinhold, Phys. Rev. Lett. 78 (1997) 2100.

[5] H. Sambe, Phys. Rev. A 7 (1973) 2203.

[6] J. Howland, Math. Ann. 207 (1974) 315.

[7] U. Peskin, N. Moiseyev, J. Chem. Phys. 99 (1993) 4590.

[8] I. Gilary, P. R. Kaprálová-Žd'ánská, N. Moiseyev, Phys. Rev. A 74 (2006) 052505.

[9] E. Balslev, J. Combes, Comm. Math. Phys. 22 (1971) 280.

[10] B. Simon, Comm. Math. Phys. 27 (1972) 1.

[11] B. Simon, Ann. Math. 97 (1973) 247.

[12] W. P. Reinhardt, Ann. Rev. Phys. Chem. 33 (1982) 223.

[13] N. Moiseyev, Phys. Rep. 302 (1998) 212.

[14] N. Moiseyev, Non-Hermitian Quantum Mechanics, Cambridge University Press, 2011.

[15] S.-I. Chu, W. P. Reinhardt, Phys. Rev. Lett. 39 (1977) 1195.

[16] G. Floquet, Ann. Sci. Ecole. Norm. Sup. 12 (1883) 47.

[17] N. Moiseyev, J. Phys. B 31 (1998) 1431.

[18] Y. Sajeev, N. Moiseyev, J. Chem. Phys. 127 (2007) 034105.

[19] U. V. Riss, H. D. Meyer, J. Phys. B 26 (1993) 4503.

[20] Y. Sajeev, V. Vysotskiy, L. S. Cederbaum, N. Moiseyev, J. Chem. Phys. 131 (2009) 211102. 
[21] Y. Sajeev, Chem. Phys. Lett. 587 (2013) 105.

[22] N. Moiseyev, P. Certain, F. Weinhold, Mol. Phys. 36 (1978) 1613.

[23] N. Moiseyev, S. Friedland, P. R. Certain, J. Chem. Phys. 74 (1981) 4739.

[24] A. Fleischer, N. Moiseyev, Phys. Rev. A 72 (2005) 032103.

[25] Y. G. Khait, A. Azenkeng, H. Wang, T. J. Dudley, M. R. Hoffmann, J. Chem. Phys. 122 (2005) 094111.

[26] Y. G. Khait, M. R. Hoffmann, Int. J. Quant. Chem. 105 (2005) 921.

[27] W. Jiang, Y. G. Khait, M. R. Hoffmann, J. Chem. Phys. 127 (2007) 164308.

[28] O. Sinanoglu, Electron Correlation in Atoms and Molecules, John Wiley and Sons, Inc., 2007, p. 237. 


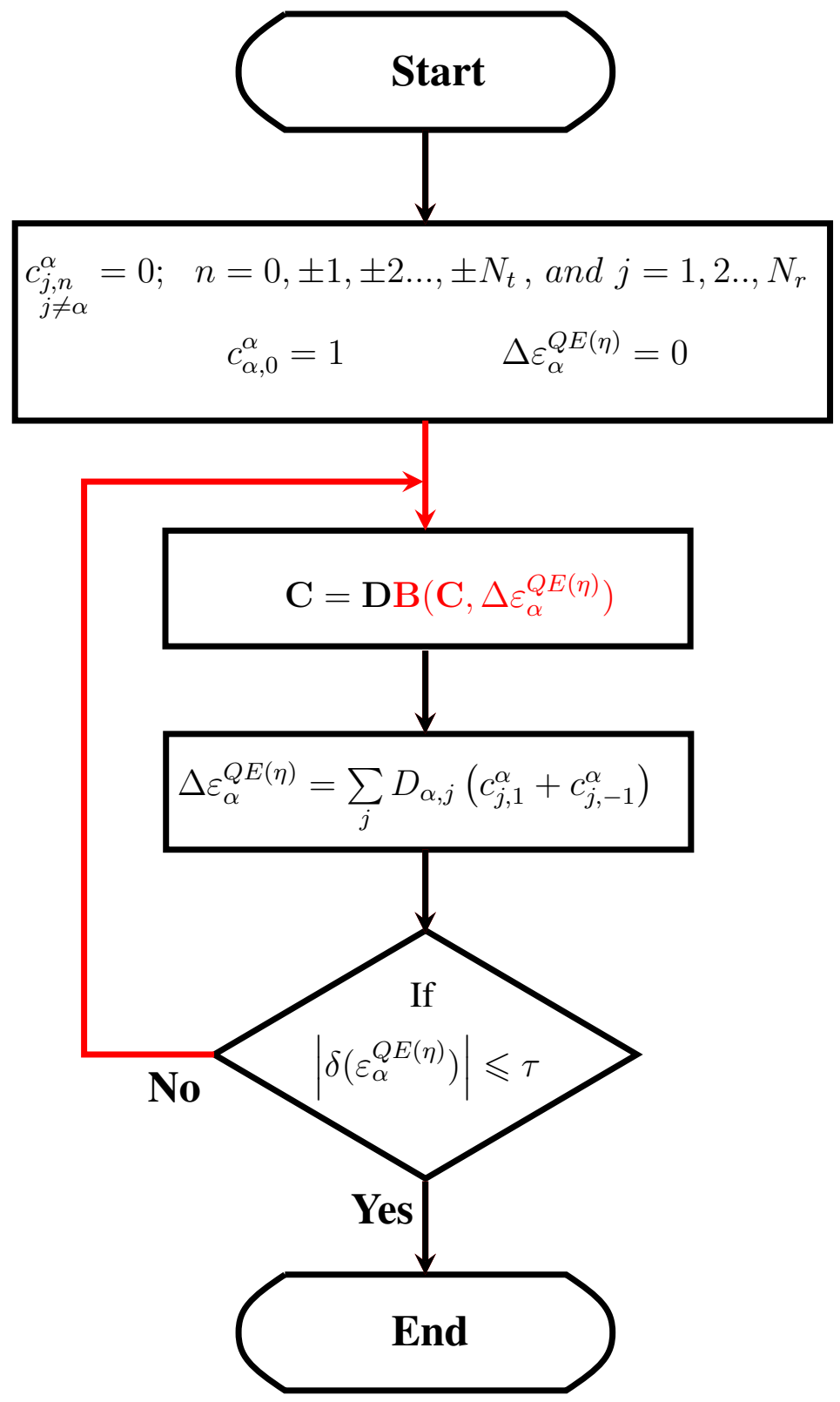

Figure 1: A simplified flow diagram for the computation of quasienergy is shown. The matrices $\mathbf{D}$ and $\mathbf{B}$ are defined in equations (21) and (22). The quantity $\delta\left(\varepsilon_{\alpha}^{Q E(\eta)}\right)$ is the difference in the quasienergy between two successive iterations and $\tau$ is the threshold for convergence. 


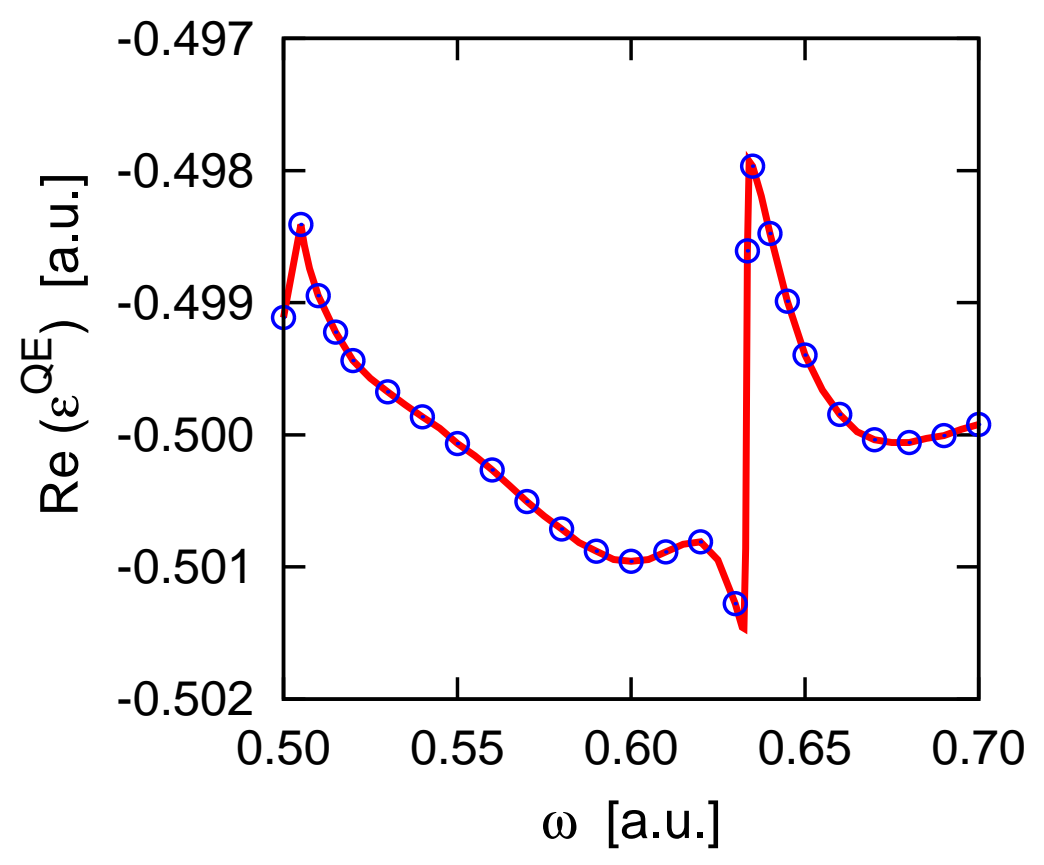

Figure 2: The position of the photoionization resonance state as a function of laser frequency is shown. The solid line represents the results from the iterative method; the circled marks represent the results from the diagonalization method. 


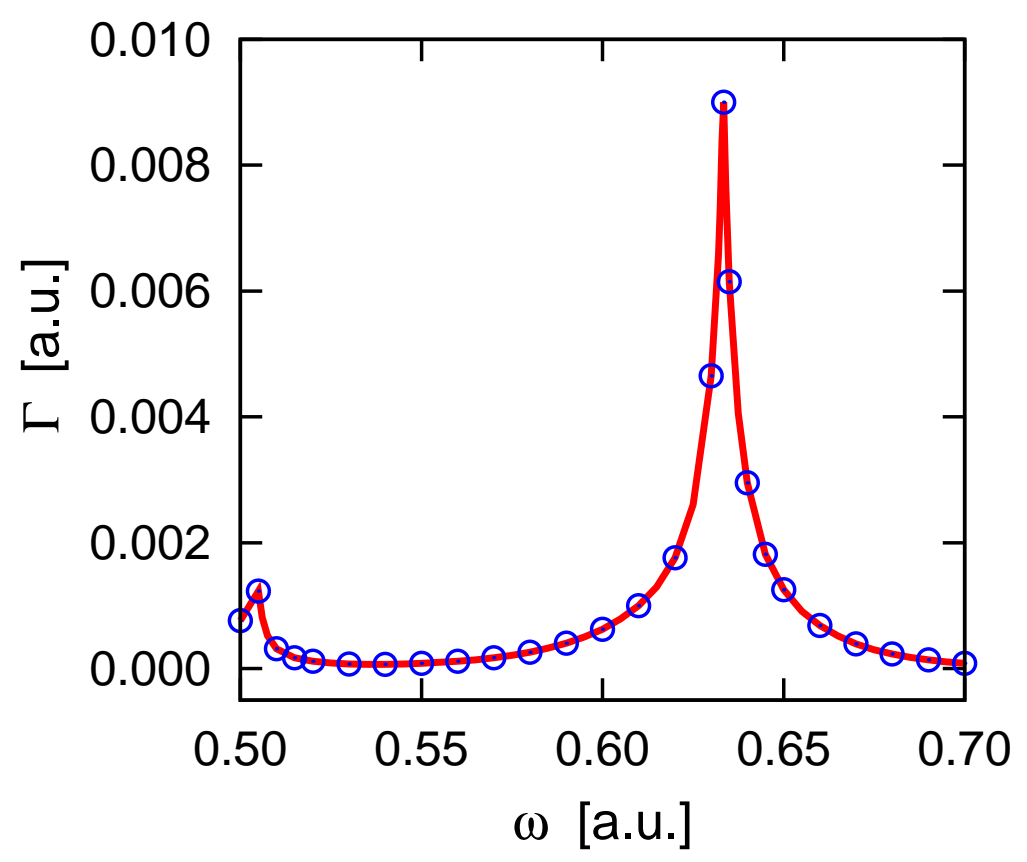

Figure 3: The width of the photoionization resonance state as a function of laser frequency is shown. The solid line represents the results from the iterative method; the circled marks represent the results from the diagonalization method. 